\title{
Influence of environmental experience on aversive conditioning in honey bees (Apis mellifera L.)
}

\author{
Timothy E. Black ${ }^{1}$, Ova FofaH ${ }^{1}$, Tugrul GiraY ${ }^{2}$, Harrington Wells ${ }^{3}$, Yves Le Conte ${ }^{4}$, \\ Charles I. ABRAMSON ${ }^{1}$ \\ ${ }^{1}$ Laboratory of Comparative Psychology and Behavioral Biology, Department of Psychology, Oklahoma State Univer- \\ sity, 116 N. Murray, Stillwater, OK 74078, USA \\ ${ }^{2}$ Department of Biology, University of Puerto Rico, Rio Piedras, Puerto Rico \\ ${ }^{3}$ Department of Biological Sciences, University of Tulsa, Tulsa, OK, USA \\ ${ }^{4}$ INRA, UR 406 Abeilles et Environnement, Avignon, France
}

Received 28 February 2018 - Revised 14 July 2018 - Accepted 8 August 2018

\begin{abstract}
Previous experience in a natural environment with a stimulus has lasting influences on honey bee behavior, as demonstrated in laboratory studies of appetitive conditioning. However, it is unknown whether the same holds true for studies of aversive conditioning. Aversive conditioning is important for insects such as honey bees to survive environmental risks. Previous experience in natural settings may lead to maladaptive behavioral patterns in bees exposed to new risks. This study presents the first examination of the effect of a visual stimulus presented in a naturalistic setting on aversive conditioning, using the shuttle box choice chamber paradigm. The present study examines both the effect of the visual stimuli, as well as differences present between the Apis mellifera subspecies of mellifera and ligustica. Results support the presence of behavioral biases based on the visual stimulus presented prior to the experimental sessions.
\end{abstract}

aversive conditioning / memory carry-over / bias / honey bee / Apis mellifera mellifera / Apis mellifera ligustica / shuttle box

\section{INTRODUCTION}

Learned associations between color or odor cues and nectar rewards have a more prominent effect on the behavior of honey bees (Apis mellifera) in new environmental contexts than was previously expected (e.g., Amaya-Márquez et al. 2014). Honey bees have been shown to reliably respond to visual cues in the form of color variation in response to appetitive conditioning (Hori et al. 2006), with some instances being observed of previous experience-altering performance on conditioning tasks (Menzel et al. 1993).

Corresponding author: C. Abramson, Charles.abramson@okstate.edu Manuscript editor: Monique Gauthier
It is, however, currently unknown how previous experience with visual cues affects performance in aversive conditioning. Here, we investigate the influence of cues learned by foraging honey bees in a subsequent shuttle box aversive-conditioning task, while also examining whether or not multiple subspecies of Apis mellifera respond to the stimuli in the same manner.

It is important to note that many of the interactions between a honey bee and visual cues are associated with floral cues. The ability of honey bees to respond to reward differences in nectar offered by alternative flower choices is well documented both in the agricultural literature (reviewed in Free 1970; Percival 1965) and in controlled experiments, where bees are known to quickly switch flower color fidelity in response to changes in rewards (e.g., Wells and Wells 1986; 
Sanderson et al. 2006; Giray et al. 2015). In fact, complete learning often occurs within just three flowers being visited at the initiation of foraging on a single trip from the hive (Menzel and Erber 1978; Menzel 1999), and is reflected in a rapid reversal in flower fidelity in the case of such reward variation (Hill et al. 1997).

The dynamic rapid learning exhibited in honey bee foragers would suggest that they approach new environments as novel situations. Nevertheless, memory carry-over appears to play a significant role in honey bee foraging behavior when approaching new situations (Chittka 1998; Cheng and Wignall 2006; Amaya-Márquez et al. 2014, 2017). This memory anachronism was clear when bees exploited a new floral landscape in the afternoon at a different geographic location from where they foraged in the morning (AmayaMárquez et al. 2014). Foragers still showed high fidelity to the flower offering the greater caloric reward in the morning patch even though flower shape, color, floral scent, time of day, and flower patch location were different from the morning flower patch. In addition, this phenomenon continued in a scenario where rewards were identical in both flower colors.

Recently, it was demonstrated that the carryover of information learned was extended to illustrate a response over a delay of several hours between morning and afternoon (AmayaMárquez et al. 2014), through overnight (Chittka 1998; Cheng and Wignall 2006), and over a stretch of several days when bees have not visited a flower patch (Amaya-Márquez et al. 2017). The fading of a memory was not a simple passive time-decay process as previously thought (Keasar et al. 1996), but rather memory remained remarkably intact without exposure to new reward differences (Amaya-Márquez et al. 2017). This is potentially due to the tendency of honey bees to be reluctant to abandon a particular feeding patch. While evidence suggests that an individual food source may be abandoned if others are readily available in the immediate area (Wells and Wells 1986; Sanderson et al. 2006; Giray et al. 2015), honey bees show remarkable consistency in returning to geographical areas that offer an abundance of food sources (Al Toufailia et al. 2013). Results collected by Al Toufailia et al. (2013) show that honey bees maintain patch fidelity, returning to many areas once abundant with food, even when individual food sources are no longer present, suggesting that while individuals may show behavioral plasticity when sources are abundant, they still maintain fidelity to particularly salient cues.

This suggests that new experiences involving difference in rewarding flowers are central for altering learned flower-reward relations in honey bees. Honey bees appear to not be able to use the removal of a cue in learning either to expect a reward or punishment (e.g., Abramson 1986; Suddendorf and Corballis 2007; Abramson et al. 2010).

Finally, expectation of a specific reward is readily apparent in honey bee behavior when rewards are altered between sequential treatments offered without a between-treatment delay (e.g., Bitterman 1976; Wells and Wells 1986; Wells et al. 1992; Hill et al. 1997; Menzel 1999; Sanderson et al. 2006), which is a reflection of "learned expectancy" well described in vertebrates (Elliott 1928; Crespi 1942). Foragers still associated a flower color initially learned to be associated with the greater nectar reward after several intervening days; bees expected a reward (Amaya-Márquez et al. 2017). Nevertheless, after days without visiting the flower patch an uncoupling of task with reward expectation seems to be supported by some studies with honey bees (Bitterman 1976; Amaya-Márquez et al. 2017). Thus, "immediacy" may be a component for honey bees to recognize differences.

In the numerous works involving honey bee behavior, several notable subspecies differences have been identified (Guzman-Novoa et al. 1996; Giannoni-Guzmán et al. 2014). While many of these differences have their basis in foraging behaviors (Cakmak et al. 2010) or types of resources harvested (Köppler et al. 2007), they have been shown to elicit behavioral differences when exposed to non-natural laboratory settings. For example, Couvillon et al. (2010) demonstrated that acquisition curves differed significantly between a common domesticated species of honey bee (Apis mellifera ligustica) and a comparable tropical hybrid species (Apis mellifera scutellata). These studies illustrate that behavioral differences 
at the subspecies level can lead to altered performance within experimental tasks. These may represent ecological and evolutionary differences of the populations (see Kence et al. 2013). In addition, these genetic subspecies differences can help in understanding the mechanisms of behavior (e.g., Rivera-Marchand et al. 2012, Giray et al. 2000).

A shuttle box, sometimes known as a choice chamber, is an apparatus where an animal moves (or "shuttles") between two or more compartments. Shuttle boxes are available for many species including ants (Abramson et al. 1982), dogs (Seligman and Maier 1967), fish (Horner et al. 1961), and rats (Warner 1932). It is one of the most popular apparatuses used to study aversiveconditioning phenomena such as place learning, escape, avoidance, and time allocation (DeCarlo and Abramson 1989). While honey bees have been shown to respond reliably to aversive conditioning based in a shuttle box paradigm (Dinges et al. 2017), it is currently unknown whether or not exposure to a cue, prior to conditioning, has any effect on an individual's performance within the shuttle box chamber. In the experiments reported here, we investigate the possible role of an experiential color bias based upon prior nonaversive experiences in aversive-conditioning situations using a shuttle box system. We assess the importance of the color an individual experiences prior to experimental conditions, as well as subspecies behavior when examining the resultant behavior within the aversive conditioning.

\section{METHODS AND MATERIALS}

\subsection{Subjects}

Research was conducted on a total of 182 bees collected from research hives maintained by the Institut National de la Research Agronomique (INRA) in Avignon, France. Subjects consisted of bees from two subspecies: Apis mellifera mellifera and Apis mellifera ligustica. Morphological assessment was used to ensure no subspecies drift between hives. All A. mellifera mellifera bees possessed the characteristic dark coloration, while all $A$. mellifera ligustica bees possessed the characteristic bright-yellow coloration.

Hives were acquired 2 months prior to experimentation and maintained in an isolated location roughly $1 \mathrm{~km}$ away from the research facility. Hives were grouped by subspecies and located approximately $1.5 \mathrm{~m}$ from one another. Subspecies were located in separate hive clusters in the same area. Clusters were maintained approximately $10 \mathrm{~m}$ apart. Each cluster consisted of three individual research hives.

Bees were collected daily over the course of 3 weeks and experienced experimental trials within $24 \mathrm{~h}$ of collection. All subjects were stored within the communal-training box with exposure to the color stimulus assigned to their group prior to the experimental trials. For each trial, an opening in the communal cage was uncovered, allowing bees to crawl into a $15-\mathrm{mL}$ Falcon tube, which were then transferred to allow the animals to enter the apparatus.

\subsection{Apparatus}

The experiments were conducted using a shuttle box (Dinges et al. 2017). The apparatus consists of two separate shuttle boxes with internal compartments measuring $135 \mathrm{~mm} \times 20 \mathrm{~mm} \times$ $5 \mathrm{~mm}$. Each box was attached to a shock grid containing $2.5 \mathrm{~mm}$ diameter pins spaced $2.5 \mathrm{~mm}$ apart. Bees touching adjacent pins would complete the circuit resulting in the administration of a shock. The boxes remained separate to ensure no social contact between animals during the experimental trials. The shock grid was connected to an external power source which provided a shock of 6.5 volts at 0.05 Amps when triggered. Visual stimuli were presented consisting of two paint swatches, one green and the other yellow. These colors were chosen due to previous research indicating that they are easily distinguished by bees in aversive-conditioning scenarios (Dinges et al. 2013) and corresponded to the colors of two of the three field-training conditions (See Figure 1).

Each shuttle box was connected to a control unit consisting of a propeller experiment controller (Varnon and Abramson 2013) and user interface. Infrared beams placed inside each shuttle box detected the locations of subjects, activated 


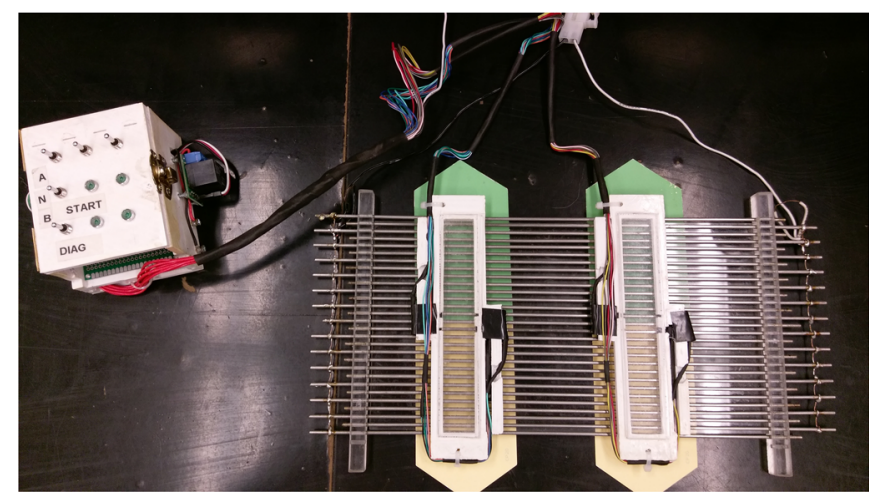

Figure 1 Shuttle box apparatus designed by Dinges et al. (2013) and the visual stimulus used for the discrimination tasks.

experiment protocols, and recorded data, in accordance with the protocol described by Dinges et al. (2017).

Feeders for training consisted of a box with a design based on the Pain cage (Pain 1966). Cages consisted of a white $50 \mathrm{~mm} \times 75 \mathrm{~mm} \times 100 \mathrm{~mm}$ polypropylene frame with two $75 \mathrm{~mm} \times 100 \mathrm{~mm}$ Plexiglas panels composing the broad side of the box. The Plexiglas panels were perforated to ensure airflow throughout the box. Sucrose lures composed of two punctured $10-\mathrm{mL}$ plastic test tubes containing a sucrose solution were inserted into each box to attract bees. For each individual color condition, a $175 \mathrm{~mm} \times 300 \mathrm{~mm}$ plastic cue board was attached to the trainer box below the sucrose lure to promote association to the color chosen. Color cues were selected such that one corresponded to each of the visual stimuli colors presented in the shuttle box (green and yellow) and a third acted as a neutral control (red), which did not appear during the shuttle box task. The colors presented within the shuttle box, green and yellow, were chosen as they are both colors encountered in the natural habitat of honey bees. Particularly, yellow is a color often associated with the flowering portion of plants, and as such, a food source for honey bees, while green corresponds to background vegetation. Red was selected as the neutral color as it corresponded to neither of the conditioned colors, and evidence suggests that honey bees have decreased, if not absent, receptors for light in the infrared or near-infrared spectra (Menzel and Blakers 1976).

\subsection{Recruitment}

In order to obtain the bees used for behavioral experiments, a series of feeder-training boxes were used to recruit foragers of each subspecies (Zhang et al. 2005, Pain 1966). Training boxes consisting of a Pain cage and visual cue board were prepared, with feeder inserts containing a $7.5 \mathrm{~mL}$ of a $50 \%$ sucrose by volume solution. The order in which the color cues for training were administered was selected using a random number generator.

During training, the box was placed outside of the target hive. Once recruitment to the feeder began, the box was relocated away from the hive by $0.5 \mathrm{~m}$ every $10 \mathrm{~min}$ until a distance of $5 \mathrm{~m}$ was reached. In each training session, the end location of the training box was varied to reduce the training effects of a specific location.

Recruitment continued until approximately 30 bees were present in the training box, at which point, the Plexiglas panel composing the top of the box was inserted. Recruitment in this manner took approximately 2 hours during which time bees became familiarized to one of the three visual cues. Bees captured in such a way were given access to the same $1.35 \mathrm{~mL}$ sucrose solution and kept exposed to the color cue until the beginning of the discrimination training. All bees were allowed at least $30 \mathrm{~min}$ from collection prior to the beginning of the experimental trials to allow for consistent exposure to the color stimulus. 


\subsection{Discrimination task}

Prior to the introduction of bees, the shuttle box apparatus was cleaned with both ethanol and distilled water to remove waste or pheromones from previous subjects. Subjects were collected from the training box using a 15-mL Falcon tube and transferred to the shuttle box. The bees were given a 5-min recovery period and experimentation began after the recovery period upon the completion of two additional criteria: That both subjects were active and detected by the apparatus, and that the bee listed as "master" was in the appropriate side of the shuttle box. After completion of these criteria, the experiment began.

Each experimental session consisted of two consecutive 5-minute trials, with the second trial starting immediately after the conclusion of the first. The two-trial system was selected, as past research has shown that this system is sufficient experience within the apparatus to reliably have the bees controlling the shock, or master bees, to restrict their activity to the safe portion of the apparatus (Dinges et al. 2017). Bees trained to one of the three colored feeders, red, green, or yellow, were introduced to the apparatus two at a time, one in each of the choice chambers. Bees were randomly assigned to one of three conditions: master, yoked, or baseline.

Baseline bees were introduced to both chambers of the shuttle box simultaneously. Bees in the baseline condition were in the shuttle box for the duration of the recovery period and both experimental trials. While in the shuttle box, baseline bees did not experience shock.

Master and yoked bees were introduced to the shuttle box in pairs, one in each compartment so that each master bee corresponded to a yoked bee. During the experimental trials, master bees controlled the onset and offset of shock. For each trial, master bees were randomly assigned to a color condition, yellow or green, corresponding to one of the two colors presented in the chamber. This color assignment corresponded to the portion of the shuttle box that would be associated with shock. When master bees entered the assigned color region of the chamber, shock was initiated. Shock terminated when the master bee exited this portion of the chamber. Yoked bees experienced shock for the same period as master bees, regardless of their position in the shuttle box.

Data were recorded on how much time each subject spend in the assigned correct half of the apparatus. For all bees, data were analyzed using percent time spend on the yellow side of the apparatus for ease of interpretation relative to the trained colors. Data were discarded for any bees which died during the experimental trials.

\subsection{Data analysis}

The data were considered to come from four experiments for statistical analysis: (I) $A$. mellifera mellifera 5-min trial 1, (II) A . mellifera mellifera 5-min trial 2, (III) A . mellifera ligustica 5-min trial 1, and (IV) A. mellifera ligustica 5min trial 2. This approach simplified interpreting results. A three-way ANOVA experimental design with interaction effects (Sall and Lehman 1996) was used to analyze data from each experiment. Each experiment was analyzed separately based on the arcsine square-root transformation of the relative frequency (proportion) of time on yellow (following Sokal and Rohlf 1995). We tested for group (i.e., master, yoked, control), priming-color (PC; i.e., green, yellow, red), correct-color (CC; i.e., green, yellow), and interaction (i.e., group $\times$ $\mathrm{CC}$; group $\times \mathrm{PC} ; \mathrm{CC} \times \mathrm{PP}$; group $\times \mathrm{PP} \times \mathrm{CC}$ ) effects.

\section{RESULTS}

\subsection{Experiments I and II}

There were significant group $\left(F_{2,80}=3.8533\right.$, $P=0.0253)$, CC $\left(F_{1,80}=12.3176, P=0.0007\right)$, and group $\times \mathrm{CC}$ interaction $\left(F_{2,80}=5.4158, P=\right.$ 0.0062 ) effects found for $A$. mellifera mellifera in the 5-min trial 1 (i.e., experiment I). The other effects were not significant (PC, $F_{2,80}=2.6353$, $P=0.0779$; group $\times \mathrm{PC}, F_{4,80}=1.3761, P=$ $0.2497 ; \mathrm{PC} \times \mathrm{CC}, F_{2,80}=0.8004, P=0.4527$; group $\times \mathrm{PC} \times \mathrm{CC}, F_{4,80}=1.5543, P=0.1946$; See Figure 2).

The results were very similar for $A$. mellifera mellifera in the 5-min trial 2 (i.e., experiment II). Although there was not a significant group effect by itself $\left(F_{2,80}=1.9558, P=0.1482\right)$, the $\mathrm{CC}$ 
(a) A.m. mellifera Baseline

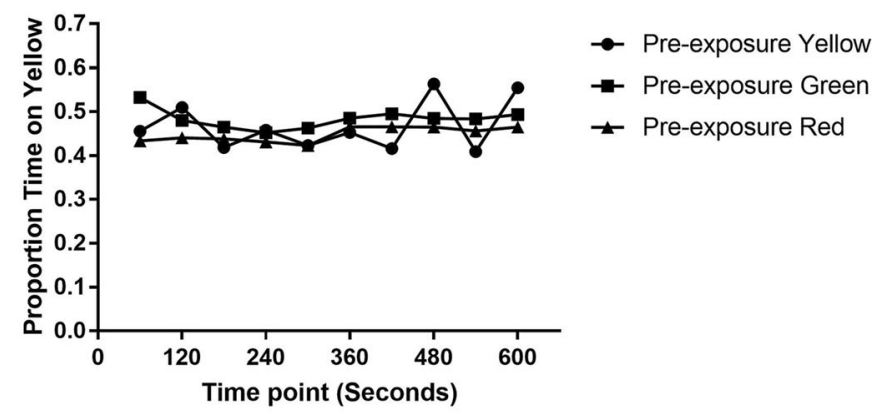

(b) A.m. mellifera Masters

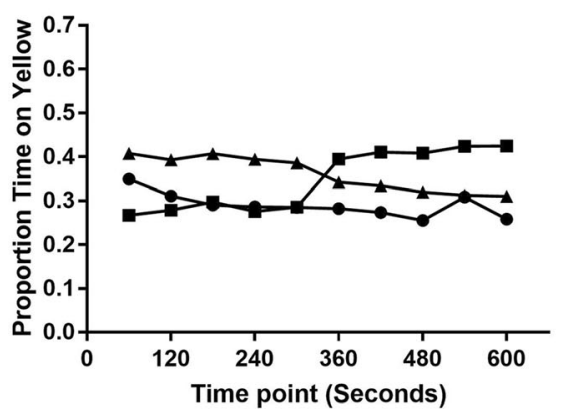

(c) A.m. mellifera Yoked

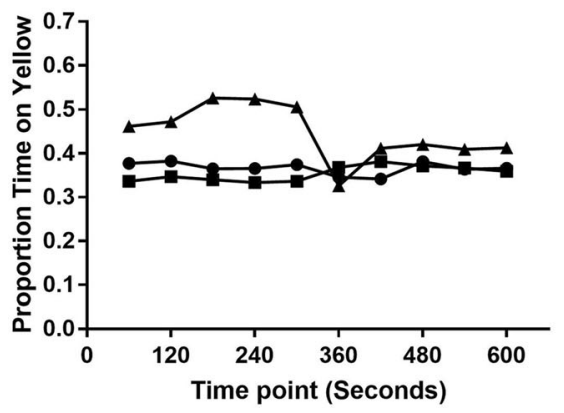

Figure 2 Graphical display of time spent on the yellow portion of the shuttle box apparatus throughout both experimental trials for all priming and learning conditions of $A$. mellifera mellifera. a Displays results for baseline bees, showing that bees trained to red $(n=10)$ did not differ from statistical chance (50\% time on yellow), while those trained to green $(n=10)$ and yellow $(n=10)$ displayed marginal preference for their trained color at the first time point, with yellow bees abandoning their preference by the second trial. b Displays results for master bees, showing that when exposed to an avoidable threatening stimuli, bees trained to red $(n=10)$, green $(n=9)$, and yellow $(n=15)$ all displayed a preference for the green portion of the apparatus, with green trained bees slowly losing their preference as the experiment progressed. c Displays results for yoked bees, showing that when exposed to unavoidable shock, bees trained to yellow $(n=12)$ and green $(n=8)$ conditions displayed a stable preference for the green portion of the apparatus. Interestingly, initially the bees trained to the red portion of the apparatus $(n=12)$ displayed no preference and developed a preference to the green portion of the apparatus by the end of the experiment. 
$\left(F_{1,80}=22.6277, P<0.0001\right)$ and group $\times \mathrm{CC}$ interaction $\left(F_{2,80}=9.8839, P=0.0001\right)$ effects were significant. In addition, the $\mathrm{PC} \times \mathrm{CC}$ interaction was significant $\left(F_{2,80}=3.3171, P=\right.$ 0.0413). The other effects were not significant $\left(\mathrm{PC}, F_{2,80}=2.2135, P=0.1160\right.$; group $\times \mathrm{PC}$, $F_{4,80}=0.8779, P=0.4810$; group $\times \mathrm{PC} \times \mathrm{CC}$, $F_{4,80}=2.1419, P=0.0832$ ).

Based on the above statistical results, we wondered whether any of the significant differences found were involved in the control or yoked groups. Thus, we performed the same analyses but using only control and yoked bees.

There were no significant effects found for the 5 -min trial 1 with only control and yoked bees in the analysis (group, $F_{1,52}=0.3110, P=0.5794$; $\mathrm{PC}, F_{2,52}=1.7296, P=0.1874 ; \mathrm{CC}, F_{1,52}=$ $1.2613, P=0.2666$; group $\times \mathrm{CC}, F_{1,52}=1.0409$, $P=0.3123$; group $\times \mathrm{PC}, F_{2,52}=2.8235, P=$ $0.0685 ; \mathrm{PC} \times \mathrm{CC}, F_{2,52}=1.3004, P=0.2811$; group $\left.\times \mathrm{PC} \times \mathrm{CC}, F_{2,52}=0.7293, P=0.4871\right)$.

Similarly, there were no significant effects found for the 5-min trial 2 with only control and yoked bees in the analysis (group, $F_{1,52}=1.1916, P=$ 0.2800 ; PC, $F_{2,52}=1.3558, P=0.2667$; CC, $F_{1,52}=2.1263, P=0.1508$; group $\times \mathrm{CC}, F_{1,52}=$ $0.8997, P=0.3473$; group $\times \mathrm{PC}, F_{2,52}=1.0255$, $P=0.3658$; Group $\times \mathrm{PC} \times \mathrm{CC}, F_{2,52}=0.1832$, $P=0.8331$ ), with the exception of a significant $\mathrm{PC}$ $\times$ CC interaction $\left(F_{2,52}=5.2328, P=0.0085\right)$.

Control and yoked bees did not show significant group, priming-color, correct-color, or interaction effect, which is to be expected since they either did not experience the punishment or their actions did not lead influence the punishment regime in any way (See Figure $2 \mathrm{a}, \mathrm{c}$ ). The master bees showed a significant $\mathrm{CC}$ effect, and in the second 5-min trial also a $\mathrm{PC} \times \mathrm{CC}$ interaction effect (See Figure 2b).

\subsection{Experiments III and IV}

The statistical analysis results for A. mellifera ligustica mirrored those for $A$. mellifera mellifera. The results for A. mellifera ligustica in the 5-min trial 1 (i.e., experiment III) did not have a significant group effect by itself $\left(F_{2,67}=\right.$ $1.4926, P=0.2322)$, but the CC $\left(F_{1,67}=6.9525\right.$, $P=0.0104)$ and group $\times \mathrm{CC}$ interaction $\left(F_{2,67}=\right.$
3.8679, $P=0.0257)$ effects were significant. All other effects were not significant $\left(\mathrm{PC}, F_{2,67}=\right.$ $0.82, P=0.4446$; group $\times \mathrm{PC}, F_{4,67}=1.8413$, $P=0.1312 ; \mathrm{PC} \times \mathrm{CC}: F_{2,67}=0.3767, P=$ 0.6876; Group $\times \mathrm{PC} \times \mathrm{CC}, F_{4,67}=1.0201, P=$ 0.4034; See Figure 3).

There were significant group $\left(F_{2,67}=\right.$ 5.1549, $P=0.0083)$, CC $\left(F_{1,67}=10.4608\right.$, $P=0.0019)$, and group $\times \mathrm{CC}$ interaction $\left(F_{2,67}=5.9490, P=0.0042\right)$ effects found for A. mellifera ligustica in the 5-min trial 2 (i.e., experiment IV). The other effects were not significant (PC, $F_{2,67}=0.5701$, $P=0.5682 ;$ group $\times \mathrm{PC}, F_{4,67}=0.9861$, $P=0.4212 ; \mathrm{PC} \times \mathrm{CC}, F_{2,67}=0.4458, P=$ 0.6422 ; group $\times \mathrm{PC} \times \mathrm{CC}, F_{4,67}=0.7359$, $P=0.5708)$.

Based on these $A$. mellifera ligustica statistical results, we again opted to test for significant differences within only the control or yoked groups. Thus, we performed the same analyses but using only control and yoked bees.

There were no significant effects found for the 5 -min trial 1 with only control and yoked bees in the analysis (group, $F_{1,44}=0.2574, P=0.6144$; PC, $F_{2,44}=0.2075, P=0.8134 ; C C, F_{1,44}=$ $0.4281, P=0.5163$; group $\times \mathrm{CC}, F_{1,44}=0.4944$, $P=0.4857$; group $\times \mathrm{PC}, F_{2,44}=0.8437, P=$ $0.4369 ; \mathrm{PC} \times \mathrm{CC}, F_{2,44}=0.3468, P=0.7089$; group $\times \mathrm{PC} \times \mathrm{CC}, \mathrm{F}_{2,44}=2.2105, P=0.1217$ ) .

Also, there were no significant effects found for the 5-min trial 2 with only control and yoked bees in the analysis (group, $F_{1,44}=0.4071, P=0.5267$; PC, $F_{2,44}=0.7134, P=0.4955 ; \mathrm{CC}, F_{1,44}=0.6034$, $P=0.4414 ;$ group $\times \mathrm{CC}, F_{1,44}=0.6368, P=$ 0.4292 ; group $\times \mathrm{PC}, F_{2,44}=0.3762, P=0.6886$; $\mathrm{PC} \times \mathrm{CC}, F_{2,44}=0.0425, P=0.9584$; group $\times \mathrm{PC}$ $\left.\times \mathrm{CC}, F_{2,44}=0.7389, P=0.4835\right)$.

The conclusions for A. mellifera ligustica are the same as those for A. mellifera mellifera, in that control and yoked bees did not show significant group, priming-color, correct-color, or interaction effect, which is to be expected since they either did not experience the punishment or their actions did not lead influence the punishment regime in any way (See Figure 3a, c). The master bees showed a significant $\mathrm{CC}$ effect, and in the second 5-min trial also a $\mathrm{PC} \times \mathrm{CC}$ interaction effect (See Figure 3b). 
(a) A.m. ligustica Baseline

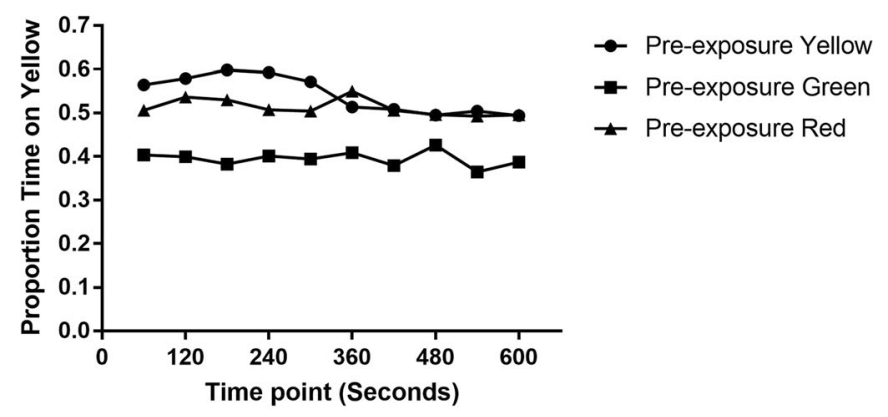

(b) A.m. ligustica Masters

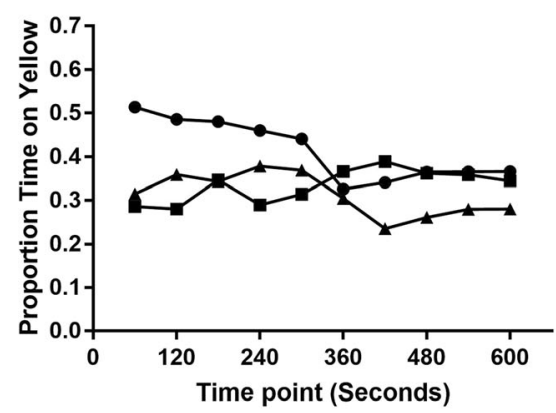

(c) A.m. ligustica Yoked

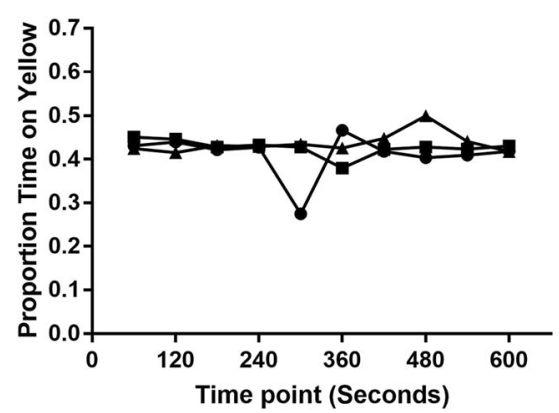

Figure 3 Graphical display of time spent on the yellow portion of the shuttle box apparatus throughout both experimental trials for all priming and learning conditions of $A$. mellifera ligustica. a Displays results for baseline bees, showing that green $(n=8)$, yellow $(n=10)$, and red $(n=10)$ trained bees all maintained a non-significant difference from statistical chance ( $50 \%$ time on yellow). However, during the first trial, it can be seen that yellow trained bees spent roughly $60 \%$ of their time in the yellow portion of the apparatus, while green trained bees only spent $40 \%$ of their time in the same side. Behavior for yellow trained bees becomes very similar to red trained bees upon entering the second trial, whereas green trained bees remain consistent with their behavior from the first trial. b Displays results for master bees, showing that when exposed to avoidable shock, bees trained to red $(n=9)$ and green $(n=10)$ conditions displayed a stable preference for the green portion of the apparatus. While green trained bees appear to slightly increase the proportion of time spent on the yellow portion of the apparatus throughout both trials, red trained bees appear to increase the proportion of time spent on the green side. Interestingly, the bees trained to yellow $(n=10)$ displayed no preference and developed a preference to the green portion of the apparatus by the end of the experiment. $\mathbf{c}$ Displays results for yoked bees, showing that when exposed on unavoidable shock, all conditions, yellow $(n=9)$, green $(n=10)$, and red $(n=9)$, expressed no preference for green or yellow portions of the apparatus. Bees trained to yellow displayed more varied behavior than bees exposed to other environmental conditions. 


\subsection{Subspecies comparison}

Based on the above analysis of data we were able to focus our comparison of the subspecies by only considering master bees. We tested for subspecies (i.e., A. mellifera ligustica, A. mellifera mellifera ), priming-color (PC; i.e., green, yellow, red), correct-color (CC; i.e., green, yellow), and interaction (i.e., subspecies $\times \mathrm{CC}$; subspecies $\times$ $\mathrm{PC}$; CC $\times$ PP; subspecies $\times \mathrm{PP} \times \mathrm{CC}$ ) effects.

The only significant effect was $\mathrm{CC}$ for the 5-min trial 1 data $\left(F_{1,51}=29.4560, P=<0.0001\right)$. The other factors were not significant (subspecies, $F_{1,51}=0.6207, P=0.4344$; PC, $F_{2,51}=2.1026$, $P=0.1326$; subspecies $\times \mathrm{CC}, F_{1,51}=.0991, P=$ 0.7542 ; subspecies $\times \mathrm{PC}, F_{2,51}=1.1714, P=$ $0.3181 ; \mathrm{PC} \times \mathrm{CC}, F_{2,51}=3.0486, P=0.0591$; subspecies $\times \mathrm{PC} \times \mathrm{CC}, F_{2,51}=0.4027, P=0.6706$ ).

The same was true for the 5-min trial 2 data. The only significant effect was CC $\left(F_{1,44}=\right.$ 47.8804, $P<0.0001)$. The other factors were not significant (subspecies, $F_{1,51}=0.5399, P=$ 0.4658 ; PC, $F_{2,51}=1.7688, P=0.1808$; subspecies $\times$ CC, $F_{1,51}=0.0936, P=0.7610$; subspecies $\times$ PC, $F_{2,51}=1.1714, P=0.3181 ; \mathrm{PC} \times$ CC, $F_{2,51}=3.0486, P=0.0562$; subspecies $\times$ $\left.\mathrm{PC} \times \mathrm{CC}, F_{2,51}=0.0333, P=0.9672\right)$.

However, the $\mathrm{PC} \times \mathrm{CC}$ interaction effect is very close to being significant for both the trail 1 $(P=0.0591)$ and trial $2(P=0.0562)$ data. This suggests that an effect of $\mathrm{PC} \times \mathrm{CC}$ interaction may in fact exist, but that it is masked by small sample sizes in the present data (See Figure 4).

\section{DISCUSSION}

The data shows that, at least for visual cues, previous experience can have a measureable effect on the behavior of subjects in a shuttle box experiment. It is worth noting that for some conditions (A. mellifera mellifera master bees trained to green), the effect is much more short-lived than others, only extending through the first of the two trials. This suggests that for this color, and similar ones, bees may need reinforcement to maintain their bias past initial results.

Other conditions, however, exhibited more marked effects of the color bias with $A$. mellifera ligustica and A. mellifera mellifera master bees trained to yellow, carrying the bias through the full two trials. This may be due to increased sensitivity to the visual wavelengths of yellow colors as opposed to green colors. This aligns with past research on the honey bee spectral range, which has noted conditioning sensitivity to $540 \mathrm{~nm}$ wavelengths, which correspond to what humans see as yellow light, but not comparable shorter wavelengths, which correspond primarily to green light (Hori et al. 2006). This data is supported by that of a concurrent study conducted at the same location, wherein A. mellifera mellifera bees showed more plasticity and switched flower color visited based on reward frequency, whereas A. mellifera ligustica did not switch (Noel et al (July 14, 2017), personal interview).

While collectively, most color conditions did initially display preferences for the side of the apparatus corresponding to their priming condition, in nearly all cases, master bees abandoned these biases by the end of the second trial. This suggests that the novel aversive conditioning may override previous appetitive conditioning. Alternatively, this result may be consistent with an effect noted by Kamin (1957), wherein research animals showed a sharp decline in conditioning recall when removed from the conditioned stimuli over relatively short periods of time. Given that animals were placed in the apparatus for $15 \mathrm{~min}$ at a time, this may account for the loss of color bias between the first and second trials.

Interestingly, there is a difference between the effects of priming colors by subspecies. These results suggest that bees of $A$. mellifera mellifera are more receptive to color stimuli, but more variable in their behavior, than those of A. mellifera ligustica, adding to the growing body of literature to suggest the presence of notable subspecies differences within domesticated honey bees (Abramson et al. 2008; Cakmak et al. 2010; Köppler et al. 2007). While the underlying cause of this difference is currently unknown, it is safe to posit that it may be due to differences in other behaviors, such as foraging habits (Cakmak et al. 2010), aggression (Abramson et al. 2008), or even available food in the native ranges of these two subspecies. Cakmak et al. (2010) observed that individuals of differing subspecies spent greater amounts of time, or a 
(a) A.m. mellifera Masters by Shock Condition

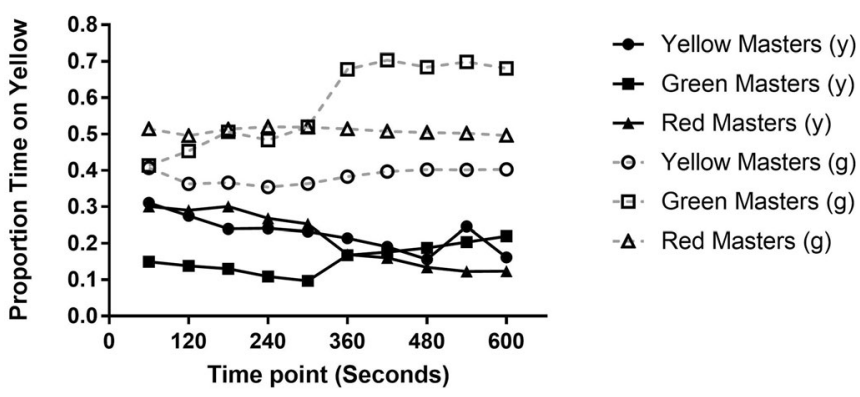

(b) A.m. ligustica Masters by Shock Condition

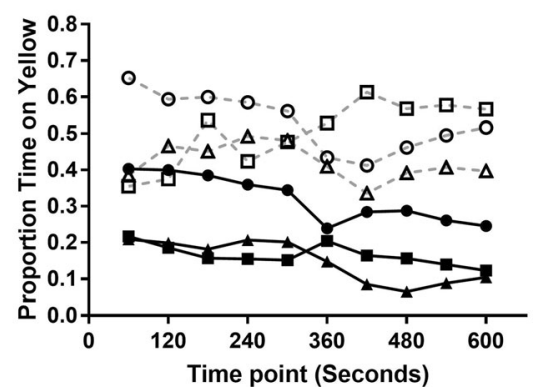

Figure 4 Graphical display of $A$. mellifera mellifera and A . mellifera ligustica master bees illustrating time spent on the yellow portion of the apparatus for all priming conditions and both shock on yellow and shock on green conditions. a Displays results for A. mellifera mellifera bees. Bees shocked on the green portion of the apparatus (dashed gray lines), on average, spent more time on the yellow portion, with red $(n=5)$ and yellow $(n=6)$ trained bees behaving consistently throughout trials. Green trained bees $(n=4)$ remained consistent with red trained bees for the first trial, and displayed an increased proportion of time spent on the yellow portion of the apparatus during the second trial. Bees shocked on the yellow portion of the apparatus (solid black lines), on average, spent more time on the green portion, with red $(n=5)$ and yellow $(n=9)$ trained bees spending more time on the green portion as the trials progressed. Green trained bees $(n=5)$, displayed a strong preference for the green side of the apparatus, spending only $10 \%$ of time on the yellow portion throughout the first trial, and increasing this proportion throughout the second trial. By the end of the second trial, all training conditions for bees shocked on yellow displayed similar behavior, spending roughly $20 \%$ time on yellow. b Displays results for A . mellifera ligustica bees. Bees shocked on the green portion of the apparatus spent, on average, more time on the yellow portion. Bees trained to red $(n=6)$ while initially expressing a slight preference for the green portion of the apparatus, maintain a pattern of behavior close to chance probability (50\% time on yellow) throughout the first trial, spending slightly less time, around $40 \%$, on yellow during the second trial. Yellow $(n=4)$ and green $(n=5)$ trained bees both displayed a preference for their trained color during the first trial, trending toward chance probability ( $50 \%$ time on yellow) by the end of the first trial. During the second trial however, green trained bees spend as much as $60 \%$ of their time on the yellow portion, while yellow trained bees remain close to chance probability. Bees shocked on the yellow portion of the apparatus, on average, spent more time on the green portion of the apparatus. Yellow trained bees $(n=5)$ displayed a strong tendency toward the yellow portion of the apparatus during the first trial, relative to other shock on yellow bees, with this effect slowly weakening throughout both trials. Red $(n=4)$ and green $(n=5)$ trained bees maintained fairly consistent behavior throughout both trials, with red trained bees gradually decreasing the proportion of time spent on the yellow portion of the apparatus, and green trained bees maintaining a strong stable preference for the green portion, at roughly $20 \%$ time spent on yellow throughout both trials.

greater number of visits, to artificial flowers of their preferred color. This behavior went so far as to include the length of time required to abandon a particular color when rewards started to diminish, and greater rewards were available elsewhere, suggesting that subspecies differ on their color 
fidelity. Of particular note is the reaction of honey bees of multiple species to dark colors when in a threatening situation. Breed et al. (2004) noted that many subspecies use cues, such as darkened colors, as a discrimination mechanism, at which point individuals will raise alarm for the colony. Regardless of the cause, it is worth to note that the two behave differently when presented with the same stimuli.

With the widespread use of this organism for both aversive and appetitive conditioning, it is important to explore potential confounds of these research methods for Apis mellifera and its collective subspecies (Abramson et al. 2011). We believe that the results of this study soundly support the presence of color biases based on food sources available for research hives. Due to this, further research must take care to account for or limit exposure to potentially confounding color stimuli when examining honey bee behavior from a naturalistic to a laboratory setting, particularly when using visual cues as conditioning stimuli. The results of these studies, indicate that honey bees, like many organisms have the potential to transfer particularly salient environmental cues to novel laboratory experiments. This, of course, carries with it the potential that these cues may present skewed or anomalous data.

Our laboratories have used the shuttle box to investigate several aspects of honey bee behavior including escape (Abramson 1986), the influence of dopamine and octopamine on avoidance learning (Agarwal et al. 2011), the role of social signals on punishment (Avalos et al. 2017), and the psychological phenomenon known as "learned helplessness" (Dinges et al. 2017). In the course of these experiments we have observed color biases.

Given the problems some laboratories face with the classical conditioning of the proboscis extension reflex in restrained honey bees (Abramson et al. 2011), we believe that the shuttle box technique will become more prominent. It is important, therefore, to examine potential sources of biases when color is used within the shuttle box. Determining the source of such biases becomes especially important as it is known that honey bees can transfer odors learned in the field to proboscis-conditioning situations in the laboratory (Gerber et al. 1996). The question naturally arises whether experience with color in the field is transferable to the shuttle box when colors are used as discriminative stimuli and if the color bias can be predicted by the colors that bees encounter in the field.

\section{ACKNOWLEDGEMENTS}

We wish to offer our gratitude to Institut National de la Research Agronomique for providing laboratory facilities, as well as access to the apiary for collection of research subjects.

Contributions TB conducted experiment, wrote drafts, and editing; OF participated in data collection and drafted methods; CA drafted intro and edited; HW edited introduction, and assisted with statistical analyses; TG editing; YL facilities and draft comments. All author read and approved the final manuscript.

\section{FUNDING INFORMATION}

Support for this research was provided by grants NSFREU (2016-1560389), NSF-HRD (1736019), and NSFOISE (2015-1545803).

Influence de l'expérience environnementale sur le conditionnement aversif chez les abeilles mellifères (Apis mellifera L.)

Conditionnement aversif / transfert de la mémoire / biais / abeille / Apis mellifera mellifera / Apis mellifera ligustica / boîte de navette

Einfluß von Umwelt-Erfahrungen auf eine aversive Konditionierung bei Honigbienen (Apis mellifera $\mathrm{L}$.)

Aversive Konditionierung / Gedächtnis-Übertragung / Bias / Honigbiene / Apis mellifera mellifera / Apis mellifera ligustica / Shuttle Box

\section{REFERENCES}

Abramson, C. I. (1986) Aversive conditioning in honey bees (Apis mellifera ). J. Comp. Psychol., 100, 108-16.

Abramson, C. I., Nolf, S. L., Mixson, T. A., \& Wells, H. (2010) Can honey bees learn the removal of a stimulus as a conditioning cue? Ethology, 116, 843-854.

Abramson, C. I., Sokolowski, M. B. C., \& Wells, H. (2011) Issues in the study of proboscis conditioning. In ( $\mathrm{F}$. Columbus, Ed.): Social Insects: Structure, Function, and Behavior. Hauppaug: Nova Science Publishers (pp. 25-49)

Abramson, C. I., Miler, J., \& Mann, D.W. (1982) An olfactory shuttle box and runway for insects. J. Mind. Behav., 3 , 151-160. 
Abramson, C. I., Mixson, T. A., Çakmak, I., Place, A. J., \& Wells, H. (2008) Pavlovian conditioning of the proboscis extension reflex in harnessed foragers using paired vs. unpaired and discrimination learning paradigms: tests for differences among honeybee subspecies in Turkey. Apidologie, 39 (4), 428-435.

Agarwal, M., Guzman, M. G., Morales-Matos, C., Del Valle Diaz, R. Abramson, C. I., and Giray, T. (2011) Dopamine and octopamine influence passive avoidance learning of honey bees in a place preference assay. PLos ONE: 6(9): e25371. doi:https://doi.org/10.1371 /journal.pone.0025371.

Al Toufailia, H., Grüter, C., \& Ratnieks, F. L. (2013). Persistence to Unrewarding Feeding Locations by Honeybee Foragers (Apis mellifera): the Effects of Experience, Resource Profitability and Season. Ethology, 119 (12), 1096-1106.

Amaya-Márquez, M., Abramson, C. I., \& Wells, H. (2017) Use of flower color-cue memory by honey bee foragers continues when rewards no longer differ between flower colors. J. Insect Behav., 30, 728-740.

Amaya-Márquez, M.,. Hill, P.S, Abramson, C. I., \& Wells, H. (2014) Honey bee location-and time-linked memory use in novel foraging situations: floral color dependency. Insects, 5, 243-269.

Avalos, A., Pérez, E., Vallejo, L., Pérez, M. E., Abramson, C. I., Giray, T. (2017) Social signals and aversive learning in honey bee drones and workers. Biol. Open 6 : 41-49 doi:https://doi.org/10.1242/bio.021543.

Bitterman, M. E. (1976) Incentive contrast in honey bees. Science, 192 , 380-382.

Breed, M. D., Guzmán-Novoa, E., \& Hunt, G. J. (2004) Defensive behavior of honey bees: organization, genetics, and comparisons with other bees. Annu. Rev. Entomol., 49(1), 271-298.

Cakmak, I., Song, D. S., Mixson, T. A., Serrano, E., Clement, M. L., Savitski, A., ... and Wells, H. (2010) Foraging response of Turkish honey bee subspecies to flower color choices and reward consistency. J. Insect Behav., 23 (2), 100-116.

Cheng, K., \& Wignall, A. E. (2006) Honeybees (Apis mellifera ) holding on to memories: response competition causes retroactive interference effects. Anim. Cogn., 9, 141-150.

Chittka, L. (1998) Sensorimotor learning in bumblebees: long-term retention and reversal training. J. Exp. Biol., 201, 515-524.

Couvillon, M. J., DeGrandi-Hoffman, G., \& Gronenberg, W. (2010) Africanized honeybees are slower learners than their European counterparts. Naturwissenschaften, 97 (2), 153-160.

Crespi, L. P. (1942) Quantitative variations of incentive and performance in the white rat. Am. J. Psychol., 55, 467-517

DeCarlo, L.T., \& Abramson, C.I. (1989) Time allocation in the carpenter ant (Componontus herculeanus). J. Comp. Psychol., 103, 389-400.
Dinges, C.W., Avalos, A., Abramson, C.I., Craig, D.P.A., Austin, Z.M., Varnon, C.A., ... \& Wells, H. (2013) Aversive conditioning in honey bees (Apis mellifera anatolica): a comparison of drones and workers. J. Exp. Biol. 216 (21), 4124-4134.

Dinges, C. W., Varnon, C. A., Cota, L. D., Slykerman, S., and Abramson, C. I. (2017) Studies of learned helplessness in honey bees (Apis mellifera ligustica). J. Exp. Psychol-Anim. L., 43 (2), 147. doi:https://doi. org/10.1037/xan0000133.

Elliott, M. H. (1928) The effect of change of reward on the maze performance of rats. Univ. CA Pub. Psychol., 4, 19-30.

Free, J. B. (1970) Insect Pollination of Crops. Academic Press, London.

Gerber, B., Geberzahn, N., F Hellstern, J. Klein, O. Kowalksy, D. Wüstenberg, \& Menzel, R. (1996) Honey bees transfer olfactory memories established during flower visits to a proboscis extension paradigm in the laboratory. Anim. Behav., 52, 1079-1085.

Giannoni-Guzmán, M.A., Avalos, A., Perez, J.M., Loperena, E.J.O., Kayım, M., Medina, J.A., ... \& Agosto-Rivera, J.L. (2014) Measuring individual locomotor rhythms in honey bees, paper wasps and other similar-sized insects. J. Exp. Biol. 217 (8), 1307-1315

Giray, T., Abramson, C. I., Chicas-Mosier, A., Brewster, T., Hayes, C., Rivera-Vega, K., Williams, M., \& Wells, H. (2015) Effect of octopamine manipulation on honeybee decision making: Reward and cost differences associated with foraging. Anim. Behav., 100, 144-150.

Giray, T., Guzmán-Novoa, E., Aron, C. W., Zelinsky, B., Fahrbach, S. E., \& Robinson, G. E. (2000) Genetic variation in worker temporal polyethism and colony defensiveness in the honey bee, Apis mellifera. Behav. Ecol. , 11 (1), 44-55.

Guzman-Novoa, E., Sanchez, A., Page Jr, R. E., \& Garcia, T. (1996) Susceptibility of European and Africanized honeybees (Apis mellifera L) and their hybrids to Varroa jacobsoni Oud. Apidologie, 27 (2), 93-103.

Hill, P. S., Wells, P. H., \& Wells, H. (1997) Spontaneous flower constancy and learning in honey bees as a function of colour. Anim. Behav., 54, 615-627

Hori, S., Takeuchi, H., Arikawa, K., Kinoshita, M., Ichikawa, N., Sasaki, M., \& Kubo, T. (2006) Associative visual learning, color discrimination, and chromatic adaptation in the harnessed honeybee Apis mellifera L. J. Comp. Physiol., 192 (7), 691-700.

Horner, J. L., Longo, N., \& Bitterman, M. E. (1961) A shuttle box for the fish and a control circuit of general applicability. Am. J. Psychol., 74, 114-120.

Kamin, L.J. (1957) The retention of an incompletely learned avoidance response. J. Comp. Physiol. Psychol. 50, 457-460

Keasar, T., Motro, U. Z. I., Shur, Y., \& Shmida, A. V. I. (1996) Overnight memory retention of foraging skills by bumblebees is imperfect. Anim. Behav., 52, 95-104.

Kence, M., Oskay, D., Giray, T., \& Kence, A. (2013) Honey bee colonies from different races show variation in 
defenses against the varroa mite in a 'common garden'. Entomol. Exp. Appl., 149 (1), 36-43.

Köppler, K., Vorwohl, G., \& Koeniger, N. (2007) Comparison of pollen spectra collected by four different subspecies of the honey bee Apis mellifera. Apidologie, 38 (4), 341-353.

Menzel, R. (1999) Memory dynamics in the honeybee. J. Comp. Physiol. A., 185, 323-340.

Menzel, R., \& Blakers, M. (1976) Colour receptors in the bee eye-morphology and spectral sensitivity. J. Comp. Physiol. A., 108(1), 11-13.

Menzel, R., \& Erber, J. (1978) Learning and memory in bees. Sci. Am., 239, 102-110.

Menzel, R., Greggers, U., \& Hammer, M. (1993) Functional organization of appetitive learning and memory in a generalist pollinator, the honey bee. In Insect learning (pp. 79-125). Springer, Boston.

Pain, J. (1966) Note technique nouveau modèle de cagettes expérimentales pour le maintien d'abeilles en captivité. Ann. Abeille, 9 (1), 71-76.

Percival, M. S. (1965) Floral biology. Pergamon Press, New York

Rivera-Marchand, B., Oskay, D., \& Giray, T. (2012) Gentle Africanized bees on an oceanic island. Evol. Appl., $5(7), 746-756$.

Sall, F.; Lehman, A. (1996) JMP IN; SAS Institute, Inc., Ducksberry Press: Belmont.
Sanderson, C. E., Orozco, B. S., Hill, P. S., \& Wells, H. (2006) Honeybee (Apis mellifera ligustica) response to differences in handling time, rewards and flower colours. Ethology, 112, 937-946.

Seligman, M. E. P., \& Maier, S. F. (1967) Failure to escape traumatic shock. J. Exp. Psychol., 74, 1-9.

Sokal, R.R.; Rohlf, F.J. (1995) Biometry; W.H. Freeman and Company: New York.

Suddendorf, T., \& Corballis, M. C. (2007) The evolution of foresight: what is mental time travel, and is it unique to humans? Behav. Brain. Sci., 30, 299-351.

Varnon, C. A., \& Abramson, C. I. (2013) The propeller experiment controller: Low-cost automation for classroom experiments in learning and behavior. Compr. Psychol., 2, 07-08.

Warner, L. H. (1932) The association span of the white rat. J. Gen. Psychol, 41, 57-89.

Wells, H., \& Wells, P. H. (1986) Optimal diet, minimal uncertainty and individual constancy in the foraging of honey bees, Apis mellifera. J. Anim. Ecol., 55, 881891.

Wells, H., Hill, P. S., \& Wells, P. H. (1992) Nectarivore foraging ecology: rewards differing in sugar types. Ecol. Entomol., 17, 280-288.

Zhang, S., Bock, F., Si, A., Tautz, J., and Srinivasan, M. V. (2005) Visual working memory in decision making by honey bees. Proc. Natl. Acad. Sci. U. S. A., 102 (14), 5250-5255. 